\title{
YIELD LOSSES CAUSED BY PLANT PARASITIC NEMATODES GRAPHICAL ESTIMATION
}

\section{Sasanelli Nicola', Toderas Ion ${ }^{2}$, Ircu-Straistaru Elena ${ }^{2,3}$, Rusu Stefan², Migunova Varvara ${ }^{4}$, Konrat Alena ${ }^{4}$}

${ }^{1}$ Institute for Sustainable Plant Protection, CNR, Bari, Italy,

2Institute of Zoology, Ministery of Education, Culture and Research, Chisinau, Republic of Moldova,

${ }^{3}$ Tiraspol State University, Chisinau, Republic of Moldova,

${ }^{4}$ All-Russian Scientific Research Institute of Fundamental and Applied Parasitology of Animals and Plants named after K.I. Skryabin, Moscow, Russia

E-mail: nicola.sasanelli@ipsp.cnr.it

https://doi.org/10.53937/9789975315975.60

Abstract: Curves for graphical estimation of yield losses caused by plant parasitic nematodes were calculated on the basis of the relationship between soil nematode population density and damage to plants as described by the Seinhort's equation $y=m$ $+(1-m) z^{(P-T)}$. By a logarithmic transformation this equation was transformed as $y=m+$ $(1-\mathrm{m}) 1.05^{[(\mathrm{P} / \mathrm{T})+1]}$ and solved considering values of tolerance limit $(T)$ and the minimum relative yield $(m)$ from the literature. The obtained curves that can be defined "Curves of nematode-pathogenicity" allow a quick and easy evaluation of yield losses for each known crop-nematode relationship.

Key words: Curves of nematode-pathogenicity, nematode population density, Seinhort's equation

\section{INTRODUCTION}

Plant parasitic nematodes can cause severe damages to numerous annual crops and the relative yield losses are correlated to the soil nematode population density at transplant or sowing. The prediction of yield losses is a fundamental prerequisite for a proper management of these pests.

The relationship between nematode density and damage to the crop is described by the Seinhorst's equation: $y=m+(1-m) z^{(P-T)}$, in which $y$ 
(relative yield) is the ratio between the yield at a given $P$ and that at $P<T$, $m=$ the minimum relative yield ( $y$ at very large $P$ ), $z=$ a constant $<1$ with $z^{-T}=1.05, P=$ initial population density and $T=$ the tolerance limit $(P$ at which no yield is lost) [32; 33; 34].

Based on this equation values of $T$ and $m$ were estimated for several nematode species on many crops (Table 1) [25]. "Tables of nematodepatogenicity" were set up, by a logarithmic manipulation of the Seinhorst's equation [26], to allow a ready and synthetic form to calculate yield losses caused by plant parasitic nematodes. This study was carried out to provide a graphic form to consult, on the base of data derived from literature, by which it is provided an easy and quick indication of yield losses for each known crop-nematode relation.

\section{MATERIALS AND METHODS}

In Table 1 are listed the fundamental parameters $T$ and $m$ of the Seinhorst's equation (1) assessed for different combination annual crop $x$ phyto-parasitic nematodes.

The construction of the "Tables of Nematode-Pathogenicity" was based on the consideration that in the Seinhorst's equation:

$z^{-T}=1.05$. (a)

Applying $\log _{1.05}$ to both terms of (a) it resulted in:

$$
\begin{gathered}
-T \log _{1.05} z=1 \text { (b) } \\
\log _{1.05} z=1 /-T(c) \\
z=1.05^{1 /-T}(d)
\end{gathered}
$$

Therefore, the expression $z^{(P-T)}$ was reported in the Seinhorst's equation as:

$$
\begin{gathered}
1.05^{[(1 /-T)(P-T)]}(\mathrm{e}) \text { from which } \\
1.05^{[(P / T / T)+1]}(\mathrm{f}) \text { and consequently: } \\
y=m+(1-m) 1.05^{[(P / T)+1]}(\mathrm{g}) .
\end{gathered}
$$

The estimation of the relative yield $(y)$ in eq. $(g)$ does not require the knowledge of $z$ reducing the unknown terms of the equation to $m$ and $T$ as previously determined in pathogenicity tests (Table 1 ) and to the initial population density $(\mathrm{Pi})$ that can be determined from analysis of soil samples. Applying eq. $(\mathrm{g})$ it resulted in the calculation of data reported in the "Tables of nematode-pathogenicity" (Table 2). 
Appropriate software for PC, Haward Graphics vs. 2.0, was used to reduce in a graphic representation the data reported in the above mentioned "Tables of Nematode-Pathogenicity" obtaining "Curves of Nematode-Pathogenicity" (Fig. 1).

\section{RESULTS AND DISCUSSION}

In the first column of the Table 2 values of the ratio $P / T$ are listed. The following columns report, for each ratio $P / T$, the values of $y$ corresponding to each value of $(1-m)$, with $m$ ranging from 0 to 0.9 . The intersection between row and column directly gives the relative yield $y$ corresponding to $P / T$ and $m$ values.

A maximum ratio $P / T$ of 75 was considered, as generally the minimum relative yield $m$ occurred just at $P / T>64$ [34].

If $P / T$ and $(1-m)$ are not entire number or multiple of 0.1 , respectively, the required estimation of the yield can be obtained by interpolation between preceding and following values of $y$ corresponding to entire values of $P / T$ or $(1-m)$. Data from Table 2 can be graphically represented by curves in which $y$ values for each ratio $P / T$ and each different $m$ value are reported. These "Curves of Nematode-Pathogenicity" could represent an alternative method to the use of "Tables of Nematode-Pathogenicity" (Fig. 1).

Table 1. Fundmental parameters $m$ and $T$ of the Seinhorst's equation for different known crop-nematode relations

\begin{tabular}{|l|l|c|c|c|}
\hline \multicolumn{1}{|c|}{ Nematode } & \multicolumn{1}{|c|}{ Crop } & $\begin{array}{c}\text { T } \\
\text { (eggs and J/ } \\
\mathbf{c m} 3 \\
\text { or g soil) }\end{array}$ & m & Authors \\
\hline Globodera pallida & Potato & 1.70 & 0.03 & {$[20]$} \\
\hline Globodera rostochiensis & Potato & 1.90 & 0.13 & {$[20]$} \\
\hline Heterodera avenae & Wheat & 1.00 & 0.60 & {$[18]$} \\
\hline Heterodera carotae & Carrot & 0.80 & 0.00 & {$[17]$} \\
\hline Heterodera ciceri & Chickpea & 1.15 & 0.00 & {$[21]$} \\
\hline & Lentil & 2.51 & 0.50 & {$[21]$} \\
\hline Heterodera cruciferae & Cabbage & 1.50 & 0.71 & {$[28]$} \\
\hline Heterodera goettingiana & Broad Bean & 0.80 & 0.10 & {$[22]$} \\
\hline
\end{tabular}




\begin{tabular}{|c|c|c|c|c|}
\hline & Pea & 0.50 & 0.00 & [22] \\
\hline & Vetch & 2.00 & 0.40 & {$[22]$} \\
\hline Heterodera schachtii & Sugarbeet & 1.80 & 0.05 & [19] \\
\hline Meloidogyne arenaria & Sweet basil & 0.15 & 0.19 & [37] \\
\hline \multirow[t]{3}{*}{ Meloidogyne artiellia } & Chickpea (spring) & 0.02 & 0.18 & [5] \\
\hline & Chickpea (winter) & 0.14 & 0.10 & {$[5]$} \\
\hline & Wheat & 0.43 & 0.10 & {$[6]$} \\
\hline Meloidogyne exigua & Coffee & 1.20 & 0.50 & {$[8]$} \\
\hline Meloidogyne hapla & Alfa-alfa & 1.60 & 0.10 & [24] \\
\hline \multirow[t]{17}{*}{ Meloidogyne incognita } & Artichoke & 1.10 & 0.00 & [7] \\
\hline & Cabbage & 0.50 & 0.05 & [28] \\
\hline & Cantaloupe & 0.19 & 0.00 & [10] \\
\hline & Cassava & 1.00 & 0.79 & [3] \\
\hline & Cowpea & 0.78 & 0.80 & [2] \\
\hline & Celery & 0.15 & 0.35 & [35] \\
\hline & Corn & 10.00 & 0.10 & [16] \\
\hline & Eggplant & 0.05 & 0.05 & [12] \\
\hline & $\begin{array}{l}\text { Grapevine (1103 Paul- } \\
\text { sen) }\end{array}$ & 1.28 & 0.85 & [30] \\
\hline & Grapevine (cv. Italia) & 0.78 & 0.60 & [30] \\
\hline & Lattuce & 0.25 & 0.38 & [1] \\
\hline & Pepper & 0.16 & 0.20 & [11] \\
\hline & Spinach & 0.25 & 0.00 & [15] \\
\hline & Sugarbeet & 1.19 & 0.1 & [9] \\
\hline & Sunflower & 1.85 & 0.25 & [27] \\
\hline & Tobacco & 2.00 & 0.00 & [10] \\
\hline & Tomato & 3.30 & 0.00 & [4] \\
\hline \multirow[t]{3}{*}{ Meloidogyne javanica } & Bean & 0.60 & 0.00 & {$[14]$} \\
\hline & Potato & 0.50 & 0.60 & [36] \\
\hline & Sunflower & 3.03 & 0.30 & [29] \\
\hline Paratrichodorus sp. & Wheat & 1.40 & 0.25 & [23] \\
\hline Pratylenchus thornei & Chickpea & 0.03 & 0.42 & [13] \\
\hline
\end{tabular}


Table 2. Table of Nematode-Pathogenicity relating crop yield (\%) to nematode population density on the base of Pi (at sowing or transplant) , T (tolerance limit of the host crop) and $m$ (minimum relative yield

\begin{tabular}{|c|c|c|c|c|c|c|c|c|c|c|}
\hline \multirow{2}{*}{$\mathbf{P} / \mathbf{T}$} & \multicolumn{10}{|c|}{$(1-m)$} \\
\hline & 1 & 0.9 & 0.8 & 0.7 & 0.6 & 0.5 & 0.4 & 0.3 & 0.2 & 0.1 \\
\hline 1 & 100.0 & 100.0 & 100.0 & 100.0 & 100.0 & 100.0 & 100.0 & 100.0 & 100.0 & 100.0 \\
\hline 2 & 95.2 & 95.9 & 97.2 & 98.5 & 99.4 & 99.8 & 100.0 & 100.0 & 100.0 & 100.0 \\
\hline 3 & 90.7 & 91.6 & 92.6 & 93.5 & 94.4 & 95.4 & 96.3 & 97.2 & 98.1 & 99.1 \\
\hline 4 & 86.4 & 87.7 & 89.1 & 90.5 & 91.8 & 93.2 & 94.6 & 95.9 & 97.3 & 98.6 \\
\hline 5 & 82.3 & $\begin{array}{l}84.0 \\
80.5\end{array}$ & 85.8 & 87.6 & 89.4 & 91.1 & 92.9 & 94.7 & 96.5 & 98.2 \\
\hline 6 & 78.4 & 80.5 & 82.7 & 84.8 & 87.0 & 89.2 & 91.3 & 93.5 & 95.7 & 97.8 \\
\hline 7 & 74.6 & $\begin{array}{c}77.2 \\
70.974 .0\end{array}$ & 79.7 & 82.2 & 84.4 & 87.3 & 89.8 & 92.4 & 94.9 & 97.5 \\
\hline 8 & 71.1 & 74.0 & 76.9 & 79.7 & 82.6 & 85.5 & 88.4 & 91.3 & 94.2 & 97.1 \\
\hline 9 & 67.7 & 70.9 & 74.1 & 77.4 & 80.6 & 83.8 & 87.1 & 90.3 & 93.5 & 96.8 \\
\hline 10 & 64.5 & 68.0 & 71.6 & 75.1 & 78.7 & 82.2 & 85.8 & 89.3 & 92.9 & 96.4 \\
\hline 11 & 61.4 & 65.3 & 69.1 & 73.0 & 76.8 & 80.7 & 84.6 & 88.4 & 92.3 & 96.1 \\
\hline 12 & 58.5 & 62.6 & 66.8 & 70.9 & 75.1 & 79.2 & 83.4 & 87.5 & 91.7 & 95.8 \\
\hline 13 & 55.7 & 60.1 & 64.5 & 69.0 & 73.4 & 77.8 & 82.3 & 86.7 & 91.1 & 95.6 \\
\hline 14 & 53.0 & 57.7 & 62.4 & 67.1 & 71.8 & 76.5 & 81.2 & 85.9 & 90.6 & 95.3 \\
\hline 15 & 50.5 & 55.5 & 60.4 & 65.4 & 70.3 & 75.3 & 80.2 & 85.2 & 90.1 & 95.1 \\
\hline 16 & 48.1 & 53.3 & 58.5 & 63.7 & 68.9 & 74.1 & 79.2 & 84.4 & 89.6 & 94.8 \\
\hline 17 & 45.8 & 51.2 & 56.6 & 62.1 & 67.5 & 72.9 & 78.3 & 83.7 & 89.2 & 94.6 \\
\hline 18 & 43.6 & 49.3 & 54.9 & 60.5 & 66.2 & 71.8 & 77.5 & 83.1 & 88.7 & 94.4 \\
\hline 19 & 41.6 & 47.4 & 53.2 & 59.1 & 64.9 & 70.8 & 76.6 & 82.5 & 88.3 & 94.2 \\
\hline 20 & 39.6 & 45.6 & 51.7 & 57.7 & 63.7 & 69.8 & 75.8 & 81.9 & 87.9 & 94.0 \\
\hline 21 & 37.7 & 43.9 & 50.2 & 56.4 & 62.6 & 68.8 & 75.1 & 81.3 & 87.5 & 93.8 \\
\hline 22 & 35.9 & 42.3 & 48.7 & 55.1 & 61.5 & 67.9 & 74.4 & 80.8 & 87.2 & 93.6 \\
\hline 23 & 34.2 & 40.8 & 47.3 & 53.9 & 60.5 & 67.1 & 73.7 & 80.3 & 86.8 & 93.4 \\
\hline 24 & 32.6 & 39.3 & 46.0 & 52.8 & 59.5 & 66.3 & 73.0 & 79.8 & 86.5 & 93.3 \\
\hline
\end{tabular}




\begin{tabular}{|c|c|c|c|c|c|c|c|c|c|c|}
\hline 25 & 31.0 & 37.9 & 44.8 & 51.7 & 58.6 & 65.5 & 72.4 & 79.3 & 86.2 & 93.1 \\
\hline 26 & 29.5 & 36.6 & 43.6 & 50.7 & 57.7 & 64.8 & 71.8 & 78.9 & 85.9 & 93.0 \\
\hline 27 & 28.1 & 35.3 & 42.5 & 49.7 & 56.9 & 64.1 & 71.2 & 78.4 & 85.6 & 92.8 \\
\hline 28 & 26.8 & 34.1 & 41.4 & 48.7 & 56.1 & 63.4 & 70.7 & 78.0 & 85.4 & 92.7 \\
\hline 29 & 25.5 & 33.0 & 40.4 & 47.9 & 55.3 & 62.8 & 70.2 & 77.7 & 85.1 & 92.6 \\
\hline 30 & 24.3 & 31.9 & 39.4 & 47.0 & 54.6 & 62.1 & 69.7 & 77.3 & 84.9 & 92.4 \\
\hline 31 & 23.1 & 30.8 & 38.5 & 46.2 & 53.9 & 61.6 & 69.3 & 76.9 & 84.6 & 92.3 \\
\hline 32 & 22.0 & 29.8 & 37.6 & 45.4 & 53.2 & 61.0 & 68.8 & 76.6 & 84.4 & 92.2 \\
\hline 33 & 21.0 & 28.9 & 36.8 & 44.7 & 52.6 & 60.5 & 68.4 & 76.3 & 84.2 & 92.1 \\
\hline 34 & 20.0 & 28.0 & $\begin{array}{l}36.0 \\
35.2 \\
\end{array}$ & 44.0 & 52.0 & 60.0 & 68.0 & 76.0 & 84.0 & 92.0 \\
\hline 35 & 19.0 & 27.1 & 35.2 & 43.3 & 51.4 & 59.5 & 67.6 & 75.7 & 83.8 & 91.9 \\
\hline 36 & 18.1 & 26.3 & 34.5 & 42.7 & 50.9 & 59.1 & 67.3 & 75.4 & 83.6 & 91.8 \\
\hline 37 & 17.3 & 25.5 & 33.8 & 42.1 & 50.4 & 58.6 & 66.9 & 75.2 & 83.5 & 91.7 \\
\hline 38 & 16.4 & 24.8 & 33.2 & 41.5 & 49.9 & 58.2 & 66.6 & 74.9 & 83.3 & 91.6 \\
\hline 39 & 15.7 & 24.1 & 32.5 & 41.0 & 49.4 & 57.8 & 66.3 & 74.7 & 83.1 & 91.6 \\
\hline 40 & 14.9 & 23.4 & 31.9 & 40.4 & 48.9 & 57.5 & 66.0 & 74.5 & 83.0 & 91.5 \\
\hline 41 & 14.2 & 22.8 & 31.4 & 39.9 & 48.5 & 57.1 & 65.7 & 74.3 & 82.8 & 91.4 \\
\hline 42 & 13.5 & 22.2 & 30.8 & 39.5 & 48.1 & 56.8 & 65.4 & 74.1 & 82.7 & 91.4 \\
\hline 43 & 12.9 & 21.6 & 30.3 & 39.0 & 47.7 & 56.4 & 65.2 & 73.9 & 82.6 & 91.3 \\
\hline 44 & 12.3 & 21.0 & 29.8 & 38.6 & 47.4 & 56.1 & 64.9 & 73.7 & 82.5 & 91.2 \\
\hline 45 & 11.7 & 20.5 & 29.3 & 38.2 & 47.0 & 55.8 & 64.7 & 73.5 & 82.3 & 91.2 \\
\hline 46 & 11.1 & 20.0 & 28.9 & 37.8 & 46.7 & 55.6 & 64.5 & 73.3 & 82.2 & 91.1 \\
\hline 47 & 10.6 & 19.5 & 28.5 & 37.4 & 46.4 & 55.3 & 64.2 & 73.2 & 82.1 & 91.1 \\
\hline 48 & 10.1 & 19.1 & 28.1 & 37.1 & 46.1 & 55.0 & 64.0 & 73.0 & 82.0 & 91.0 \\
\hline 49 & 9.6 & 18.7 & 27.7 & 36.7 & 45.8 & 54.8 & 63.8 & 72.9 & 81.9 & 91.0 \\
\hline 50 & 9.2 & 18.2 & 27.3 & 36.4 & 45.5 & 54.6 & 63.7 & 72.7 & 81.8 & 90.9 \\
\hline 51 & 8.7 & 17.8 & 27.0 & 36.1 & 45.2 & 54.4 & 63.5 & 72.6 & 81.7 & 90.9 \\
\hline 52 & 8.3 & 17.5 & 26.6 & 35.8 & 45.0 & 54.2 & 63.3 & 72.5 & 81.7 & 90.8 \\
\hline 53 & 7.9 & 17.1 & 26.3 & 35.5 & 44.7 & 54.0 & 63.2 & 72.4 & 81.6 & 90.8 \\
\hline 54 & 7.5 & 16.8 & 26.0 & 35.3 & 44.5 & 53.8 & 63.0 & 72.3 & 81.5 & 90.8 \\
\hline
\end{tabular}


Continuation of table 2.

\begin{tabular}{|c|c|c|c|c|c|c|c|c|c|c|}
\hline \multirow{2}{*}{$\mathrm{P} / \mathrm{T}$} & \multicolumn{10}{|c|}{$(1-m)$} \\
\hline & 1 & 0.9 & 0.8 & 0.7 & 0.6 & 0.5 & 0.4 & 0.3 & 0.2 & 0.1 \\
\hline 55 & 7.2 & 16.5 & 25.7 & 35.0 & 44.3 & 53.6 & 62.9 & 72.2 & 81.4 & 90.7 \\
\hline 56 & 6.8 & 16.1 & 25.5 & 34.8 & 44.1 & 53.4 & 62.7 & 72.0 & 81.4 & 90.7 \\
\hline 57 & 6.5 & 15.9 & 25.2 & 34.6 & 43.9 & 53.3 & 62.6 & 72.0 & 81.3 & 90.7 \\
\hline 58 & 6.2 & 15.6 & 25.0 & 34.3 & 43.7 & 53.1 & 62.5 & 71.9 & 81.2 & 90.6 \\
\hline 59 & 5.9 & 15.3 & 24.7 & 34.1 & 43.5 & 53.0 & 62.4 & 71.8 & 81.2 & 90.6 \\
\hline 60 & 5.6 & 15.1 & 24.5 & 33.9 & 43.4 & 52.8 & 62.2 & 71.7 & 81.1 & 90.6 \\
\hline 61 & 5.4 & 14.8 & 24.3 & 33.7 & 43.2 & 52.7 & 62.1 & 71.6 & 81.1 & 90.5 \\
\hline 62 & 5.1 & 14.6 & 24.1 & 33.6 & 43.1 & 52.5 & 62.0 & 71.5 & 81.0 & 90.5 \\
\hline 63 & 4.9 & 14.4 & 23.9 & 33.4 & 42.9 & 52.4 & 61.9 & 71.5 & 81.0 & 90.5 \\
\hline 64 & 4.6 & 14.2 & 23.7 & 33.2 & 42.8 & 52.3 & 61.8 & 71.4 & 80.9 & 90.5 \\
\hline 65 & 4.4 & 14.0 & 23.5 & 33.1 & 42.6 & 52.2 & 61.8 & 71.3 & 80.9 & 90.4 \\
\hline 66 & 4.2 & 13.8 & 23.4 & 32.9 & 42.5 & 52.1 & 61.7 & 71.3 & 80.8 & 90.4 \\
\hline 67 & 4.0 & 13.6 & 23.2 & 32.8 & 42.4 & 52.0 & 61.6 & 71.2 & 80.8 & 90.4 \\
\hline 68 & 3.8 & 13.4 & 23.0 & 32.7 & 42.3 & 51.9 & 61.5 & 71.1 & 80.8 & 90.4 \\
\hline 69 & 3.6 & 13.3 & 22.9 & 32.5 & 42.2 & 51.8 & 61.4 & 71.1 & 80.7 & 90.4 \\
\hline 70 & 3.5 & 13.1 & 22.8 & 32.4 & 42.1 & 51.7 & 61.4 & 71.0 & 80.7 & 90.3 \\
\hline 71 & 3.3 & 13.0 & 22.6 & 32.3 & 42.0 & 51.6 & 61.3 & 71.0 & 80.7 & 90.3 \\
\hline 72 & 3.1 & 12.8 & 22.5 & 32.2 & 41.9 & 51.6 & 61.3 & 70.9 & 80.6 & 90.3 \\
\hline 73 & 3.0 & 12.7 & 22.4 & 32.1 & 41.8 & 51.5 & 61.2 & 70.9 & 80.6 & 90.3 \\
\hline 74 & 2.8 & 12.6 & 22.3 & 32.0 & 41.7 & 51.4 & 61.1 & 70.9 & 80.6 & 90.3 \\
\hline 75 & 2.7 & 12.4 & 22.2 & 31.9 & 41.6 & 51.4 & 61.1 & 70.8 & 80.5 & 90.3 \\
\hline
\end{tabular}

Independently from the proposed methods of estimation of the relative yield $y$ (analytically as in the "Table of Nematode-Pathogenicity" or graphically as in the "Curve of Nematode-Pathogenicity") the percent yield loss (Yl\%) is obtained by the difference to $100: \mathrm{YI} \%=100-y$. 


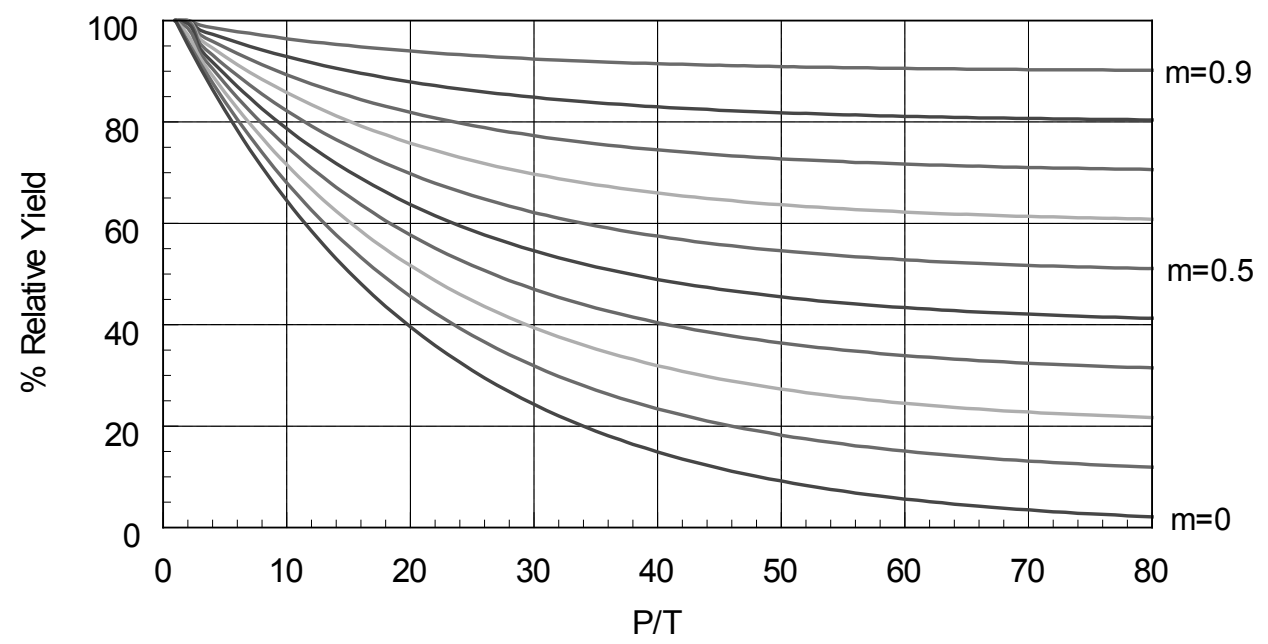

Figure 1. Curves of Nematode-Pathogenicity for an easy and quick estimation of yield losses

\section{CONCLUSIONS}

"Curves of nematode-pathogenicity" allow a quick and easy evaluation of yield losses for each known crop-nematode relationship allowing an appropriate management of these pests and decisions of agricultural producers.

If the cost of nematode control $\left(K_{c}\right)$ is lower than the value of yield losses $(\mathrm{YI})(\mathrm{Kc}<\mathrm{YI})$ it is convenient to control nematodes. On the contrary if $\mathrm{Kc}>\mathrm{Yl}$, the agricultural producer can choose a different crop (no hosts of the nematode) to improve farm economic results.

\section{BIBLIOGRAPHY}

1. Asuaje L., Jimenez A., Jimenez-Perez, Crozzoli R., 2004. Efecto del nematode agallador, Meloidogyne incognita, sobre el crecimiento de tres cultivares de lechuga. Fitopatologia Venezolana, 17(1): 2-5.

2. Crozzoli R., Greco N., Suarez A., Rivas D., 1997. Patogenicidad del nematode agallador, Meloidogyne incognita, en cultivares de Phaseolus vulgaris y Vigna unguiculata. Nematropica, 27:61-67.

3. Crozzoli R., Parra N., 1999. Patogenicidad del nematode agallador, 
Meloidogyne incognita, en yuca (Manihot esculenta). Nematologia mediterranea, 27:95-100.

4. Di Vito M., Ekanayake H.R.M.K., 1983. Relationship between population densities of Meloidogyne incognita and growth of resistant and susceptible tomato. Nematolologia mediterranea, 11:151-155.

5. Di Vito M., Greco N., 1988a. The relationship between initial population density of Meloidogyne artiellia and yield of winter and spring chickpea. Nematolologia mediterranea, 16:163-166.

6. Di Vito M., Greco N., 1988b. Effect of population densities of Meloidogyne artiellia on yield of wheat. Nematolologia mediterranea, 16:167-169.

7. Di Vito M. and Zaccheo G., 1991. Population density of root-knot nematode, Meloidogyne incognita and growth of artichoke (Cynara scolymus). Adv. Hort. Sci., 5:81-82.

8. Di Vito M., Crozzoli R., Vovlas N., 2000. Pathogenicity of Meloidogyne exigua on coffee (Coffea arabica L.) in pots. Nematropica, 30(1):55-61.

9. Di Vito M., Greco N., Carella A., 1981. Relationship between population densities of Meloidogyne incognita and yield of sugarbeet and tomato. Nematolologia mediterranea, 9:99-103.

10. Di Vito M., Greco N., Carella A., 1983. The effect of population densities of Meloidogyne incognita on the yield of cantaloupe and tobacco. Nematolologia mediterranea, 11:169-174.

11. Di Vito M., Greco N., Carella A., 1985. Population densities of Meloidogyne incognita and yield of Capsicum annuum. J.Nematol., 17:45-49.

12. Di Vito M., Greco N., Carella A., 1986. Effect of Meloidogyne incognita and the importance of the inoculum on the yield of eggplant. Journal of Nematology, 18:487-490.

13. Di Vito M., Greco N., Saxena M.C., 1992. Pathogenicity of Pratylenchus thornei on chickpea in Syria. Nematolologia mediterranea, 20:71-73.

14. Di Vito M., Parisi B., Catalano F., 2007. Pathogenicity of Meloidogyne javanica on common bean (Phaseolus vulgaris L.). Nematropica 37:339344.

15. Di Vito M., Vovlas N., Castillo P., 2004. Host-parasite relationship of Meloidogyne incognita on spinach. Plant Pathology, 53:508-514. Doi: 10.1046/j.0032-0862.2004.01053.x 
16. Di Vito M., Vovlas N., Inserra R.N., 1980. Influence of Meloidogyne incognita on growth of corn in pots. Plant Disease, 64:1025-1026.

17. Greco N., Brandonisio A., 1980. Relationship between Heterodera carotae and carrot yield. Nematologica, 26:497-500.

18. Greco N., Brandonisio A., 1987. Investigations on Heterodera avenae in Italy. Nematolologia mediterranea, 15:225-234.

19. Greco N., Brandonisio A., De Marinis G., 1982a. Tolerance limit of the sugarbeet to Heterodera schachtii. Journal of Nematology, 14:199-202.

20. Greco N., Di Vito M., Brandonisio A., Giordano I., De Marinis G., 1982b. The effect of Globodera pallida and G. rostochiensis on potato yield. Nematologica, 28:379-386.

21. Greco N., Di Vito M., Saxena M.C., Reddy M.V., 1988. Effect of Heterodera ciceri on yield of chickpea and lentil and development of this nematode on chickpea in Syria. Nematologica, 34: 98-114.

22. Greco N., Ferris H., Brandonisio A., 1991. Effect of Heterodera goettingiana population densities on the yield of pea, broad bean and vetch. Revue de Nematologie, 14:619-624.

23. Inserra R.N., Di Vito M., Vovlas N., Seinhorst J.W., 1983a. Relationship between Paratrichodorus sp. density and growth of wheat in pots. Journal of Nematology, 15:79-87.

24. Inserra R.N., O'Bannon J.H., Di Vito M., Ferris H., 1983b. Response of two alfalfa cultivars to Meloidogyne hapla. Journal of Nematology, 15:644646.

25. Lamberti F., Greco N., 1989. Perdite di produzione causate da nematodi fitoparassiti. Informatore Fitopatologico, 39 (9): 35-39

26. Sasanelli N., 1994. Tables of Nematode-Pathogenicity. Nematologia mediterranea, 22:153-157

27. Sasanelli N., Di Vito M., 1992. The effect of Meloidogyne incognita on growth of sunflower in pots. Nematologia mediterranea, 20:9-12.

28. Sasanelli N., Di Vito M. and Zacheo G., 1992a. Population densities of Meloidogyne incognita and growth of cabbage in pots. Nematologia mediterranea, 20:21-23.

29. Sasanelli N., Vovlas N., D'Addabbo T., 1992b. Influence of Meloidogyne javanica on growth of sunflower. Afro-Asian Journal of Nematology, 2:84- 
88.

30. Sasanelli N., D'Addabbo T., Liskova M., 2006. Influence of the rootknot nematode Meloidogyne incognita r.1 on growth of grapevine. Helminthologia, 43(3): 168-170. DOI 10.2478/s11687-006-0031-z

31. Sasanelli N., Vovlas N., Trisciuzzi N., Cantalapietra-Navarrete C., PalomaresRius J.E., Castillo P., 2013. Pathogenicity and Host-Parasite Relationship of Heterodera cruciferae in Cabbage. Plant Disease, 97: 333-338. DOI: 10.1094/PDIS-07-12-0699-RE

32. Seinhorst J.W., 1965. The relationship between nematode density and damage to plants. Nematologica, 11:137-154

33. Seinhorst J.W., 1979. Nematodes and growth of plants: formulation of the nematode-plant system. In: Root-knot nematodes (Meloidogyne species) Systematics, Biology and Control. (F. Lamberti and C. E. Taylor Eds), Academic Press, London, pp. 231-256

34. Seinhorst J.W., 1986. Effect of nematode attack on the growth and yield of crop plants. In: "Cyst Nematodes". (F. Lamberti and C. E. Taylor Eds), Plenum Press, New York, London, pp. 191-209

35. Vovlas N., Lucarelli G., Sasanelli N., Troccoli A., Papajova I., Palomares-Rius J.E., Castillo P., 2008. Pathogenicity and host-parasite relathionships of the root-knot nematode Meloidogyne incognita on celery. Plant Pathology, 981-987. Doi: 10.1111/j.1365-3059.2008.01843.x

36. Vovlas N., Mifsud D., Landa B.B., Castillo P., 2005. Pathogenicity of the rootknot nematode Meloidogyne javanica on potato. Plant Pathology, 54:657664. Doi: 10.1111/j.1365-3059.2005.01244.x

37. Vovlas N., Troccoli A., Minuto A., Bruzzone C., Sasanelli N., Castillo P., 2008. Pathogenicity and Host-Parasite Relationships of Meloidogyne arenaria in Sweet Basil. Plant Disease, 92 (9): 1329-1335. doi:10.1094/PDIS-92-91329 